\title{
Determinants of biomarkers of bone turnover in a sample of young European adults
}

\author{
A. Lucey ${ }^{1}$, G. Paschos $^{1}$, K. D. Cashman ${ }^{1,2}$, J. A. Martínéz ${ }^{3}$, I. Thorsdottir ${ }^{4}$ and M. Kiely ${ }^{1}$ \\ ${ }^{1}$ Department of Food \& Nutritional Sciences and ${ }^{2}$ Department of Medicine, University College Cork, Cork, Republic of \\ Ireland, ${ }^{3}$ Department of Physiology \& Nutrition, University of Navarra, Navarra, Spain and ${ }^{4}$ Unit for Nutrition Research, \\ Landspitali University Hospital, Reykjavik, Iceland
}

Bone-turnover markers (BTM) reflect whole-body rates of bone resorption and bone formation ${ }^{(1)}$. In order to interpret BTM data appropriately not only should the analytical performance of the assay be considered but also sources of pre-analytical variability ${ }^{(2)}$. Preanalytical variability arises from both personal characteristics of the study population such as age, gender, ethnicity and menopausal status, and modifiable variables such as dietary factors and the timing and conditions of sample collection ${ }^{(3)}$. The current analysis investigates determinants of BTM in European men and women aged 30-43 years.

Lifestyle data, biomarkers of metabolic health, nutrient intakes and fasting levels of the biomarkers of bone formation (serum osteocalcin (OC) and bone-specific alkaline phosphatase (BAP)) and bone resorption (serum C-telopeptide of type 1 collagen (CTx) and urinary $\mathrm{N}$-telopeptide of type I collagen (NTx)) were collected between October 2004 and March 2005 in 173 overweight adults (BMI $27.5-$ $32.5 \mathrm{~kg} / \mathrm{m}^{2}$ ) from Iceland, Spain and Ireland. The lifestyle and health variables were collected as previously described ${ }^{(4)}$. Dietary intakes were measured using a $2 \mathrm{~d}$ weighed-food record. Multiple linear regression models examined relationships between lifestyle, health and dietary variables and BTM. Significant associations are summarised in the Table.

\begin{tabular}{|c|c|c|c|c|c|c|c|}
\hline & $\beta$ & $\mathrm{SE}$ & $P$ & & $\beta$ & SE & $P$ \\
\hline Serum OC $(\mathrm{ng} / \mathrm{ml})$ & & & & Urinary NTx (nм BCE/mm creatinine) & & & \\
\hline Age (years) & -0.272 & 0.198 & 0.004 & Country & -0.448 & 0.047 & $<0.001$ \\
\hline Fasting serum 25(OH)D $(\mathrm{nmol} / \mathrm{l})$ & -0.214 & 0.014 & 0.025 & Age (years) & -0.272 & 0.198 & 0.004 \\
\hline Fasting serum LDL-cholesterol $(\mathrm{mg} / \mathrm{dl})$ & -0.343 & 0.019 & 0.003 & Vitamin $\mathrm{C}$ intake $(\mathrm{mg} / \mathrm{d})$ & 0.189 & 0.049 & 0.046 \\
\hline Vitamin $\mathrm{C}$ intake $(\mathrm{mg} / \mathrm{d})$ & 0.475 & 0.019 & $<0.001$ & Fat intake $(\mathrm{g} / \mathrm{d})$ & 0.456 & 0.062 & $<0.001$ \\
\hline Fibre intake $(\mathrm{g} / \mathrm{d})$ & -0.316 & 0.02 & 0.011 & Vitamin $B_{12}$ intake $(\mu \mathrm{g} / \mathrm{d})$ & 0.272 & 0.063 & 0.027 \\
\hline $\mathrm{K}$ intake (mg/d) & -0.247 & 0.017 & 0.030 & Serum CTx $(\mathrm{ng} / \mathrm{ml})$ & & & \\
\hline Serum BAP (U/l) & & & & Country & -0.370 & 0.037 & $<0.001$ \\
\hline Gender & -0.448 & 0.017 & $<0.001$ & Gender & -0.361 & 0.042 & $<0.001$ \\
\hline Fasting serum leptin (ng/ml) & -0.225 & 0.020 & 0.005 & Smoking status & 0.238 & 0.025 & 0.011 \\
\hline Fasting serum insulin $(\mathrm{mU} / \mathrm{l})$ & -0.159 & 0.018 & 0.024 & Diastolic blood pressure $(\mathrm{mmHg})$ & -0.242 & 0.043 & 0.011 \\
\hline \multirow[t]{4}{*}{ Folic acid intake $(\mu \mathrm{g} / \mathrm{d})$} & 0.137 & 0.018 & 0.051 & Fasting serum cortisol $(\mu \mathrm{g} / \mathrm{dl})$ & 0.238 & 0.044 & 0.008 \\
\hline & & & & Vitamin C intake $(\mathrm{mg} / \mathrm{d})$ & 0.202 & 0.038 & 0.020 \\
\hline & & & & Fat intake $(\mathrm{g} / \mathrm{d})$ & 0.520 & 0.070 & 0.002 \\
\hline & & & & Vitamin $B_{12}$ intake $(\mu \mathrm{g} / \mathrm{d})$ & 0.205 & 0.041 & 0.029 \\
\hline
\end{tabular}

Alcohol intake, BMI (possibly because of the narrow range included) and systolic blood pressure did not appear to influence levels of BTM. Factors such as age, gender, country of origin and smoking status were significant determinants of BTM levels and should be accounted for when interpreting bone marker data. In addition, these data indicate that non-bone-related physiological indices, e.g. fasting insulin, may influence BTM. In addition, the influence of habitual diet on circulating levels of BTM requires further investigation.

The YOUNG study (coordinator Professor Inga Thorsdottir) is part of the SEAFOODplus Integrated Project (coordinator Professor Torger Børresen), which is funded by the EC through the 6th Framework Programme Contract no. FOOD-CT-2004-506359.

1. de Papps AE, Bone HG, Caulfield MP et al. (2007) Bone 40, 1222-1230.

2. Glover SJ, Garnero P, Naylor K et al. (2008) Bone 42, 623-630.

3. Hannon R \& Eastell R (2000) Osteoporos Int 11, S30-S44.

4. Thorsdottir I, Thomasson H, Gunnarsdottir I et al. (2007) Int J Obes 31, 1560-1566. 